\title{
Unexpected cleavage of an ester group by hydrogenolysis reactions of Passerini subtrates.
}

\section{Thaíssa Pasquali F. Rosalba (PG)a , Angélica de Fátima S. Barreto (PG) and Carlos Kleber Z. Andrade (PQ) ${ }^{\star a}$}

\author{
a Laboratório de Química Metodológica e Orgânica Sintética (LaQMOS), Instituto de Química, Universidade \\ de Brasília, CP-4478, 70910-970 Brasília-DF, Brasil \\ *ckleber@unb.br
}

Keywords: Passerini reaction and 2,5-diketomopholines.

\section{INTRODUCTION}

In recent years, the Passerini reaction has become a versatile tool in combinatorial synthesis and is experiencing a growing interest due to its efficiency in the synthesis of a variety of products. ${ }^{1}$ The Passerini reaction is a three-component condensation (P-3CR) between an isocyanide, a carbonyl compound and carboxylic acid that yields a-acyloxy carboxyamides. ${ }^{2}$

In the course of our research on multicomponents reactions we came across an unexpected cleavage of the ester function of several Passerini substrates which is dependent on the experimental conditions employed.

\section{RESULTS AND DISCUSSION}

Using a protocol previously established by our group ${ }^{3}$, several $\alpha$-acyloxy carboxyamides were prepared (Scheme 1). Aliphatic, aromatic and heteroaromatic aldehydes $\mathbf{1}$ were reacted with methyl/ethyl isocyanoacetate $\mathbf{2}$ and Cbz-glycine $\mathbf{3}$ (Scheme 1). The three components were subjected to microwave irradiation for $4 \min \left(60{ }^{\circ} \mathrm{C}, 40 \mathrm{~W}\right)$ to give products $4 a-f$ in moderate to good yields.

When compounds 4a-f were subjected to a hydrogenolysis reaction in the presence of $\mathrm{Pd} / \mathrm{C}$ as catalyst under hydrogen atmosphere using methanol as solvent, the expected products 5a-f were not obtained. Instead, compounds $6 \mathbf{a - c}$ or $7 \mathbf{a}-\mathbf{c}$ were isolated in near quantitative yields in a very clean process. Interestingly and most surprising is the removal of the hydroxyl groups for substrates $6 \mathbf{b}$ and $\mathbf{6 c}$ under longer reaction periods. In these cases, compounds $\mathbf{7 b}, \mathbf{c}$ could be obtained when the reaction was left for a short time ( $\leq 5 \mathrm{~min})$.

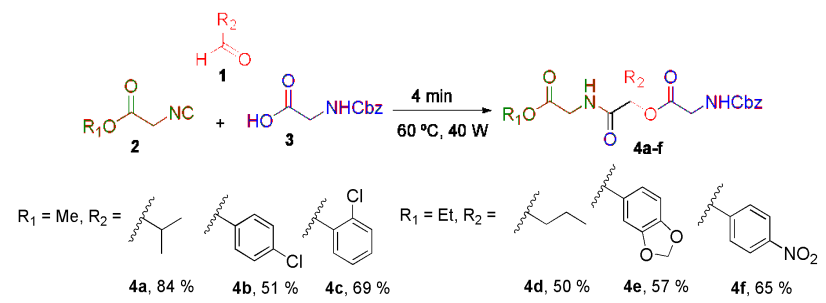

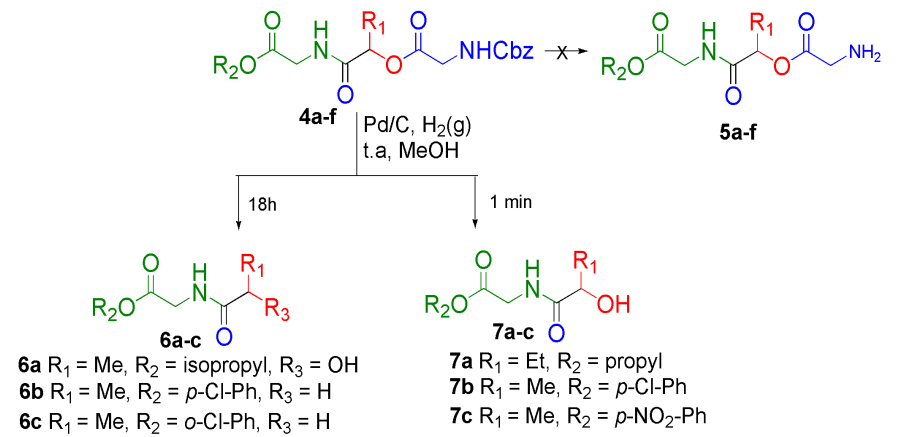

Scheme 2. Study of the hydrogenolysis reaction.

Substrates 7a-c can be used in the synthesis of 2,5diketomorpholines derivatives (8) and this cyclization step is in progress in our laboratory (Scheme 3 ).<smiles>[R]OC(=O)CNC(=O)C([R2])O</smiles>

Scheme 3. 2,5-diketomorpholines synthesis.

\section{CONCLUSION}

The unexpected results so far obtained in the hydrogenolysis of Passerini substrates can be used as an attractive methodology in the synthesis of 2,5diketomorpholines.

\section{ACKNOWLEDGEMENTS}

IQ-UnB, Capes, CNPq and FINEP-CTINFRA no 0970/01.

\section{REFERENCES}

${ }^{1}$ Domling, A.; Ugi, I. Angew. Chem. Int. Ed. 2000, 39, 3169.

2 Passerini, M.; Gazz. Chim. Ital. 1921, 5, 126.

${ }^{3}$ Barreto, A .F. S.; Vercillo, O. E..; Andrade, C. K. Z. J. Braz. Chem. Soc. 2011, 22, 462 .

Scheme 1. Synthesis of $\alpha$-acyloxy carboxyamides. 